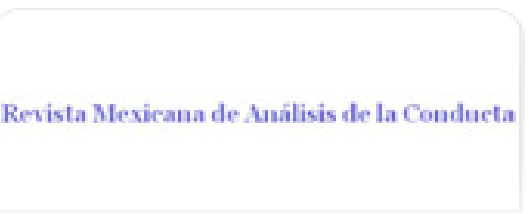

Revista Mexicana de Análisis de la Conducta ISSN: 0185-4534

editora@rmac-mx.org

Sociedad Mexicana de Análisis de la Conducta

México

ESCOBAR, ROGELIO; PÉREZ-HERRERA, CARLOS A. PALANCA RETRÁCTIL DE BAJO COSTO PARA INTERFACES CON PUERTO PARALELO Y VISUAL BASIC®

Revista Mexicana de Análisis de la Conducta, vol. 39, núm. 3, diciembre-, 2013, pp. 56-64 Sociedad Mexicana de Análisis de la Conducta Distrito Federal, México

Disponible en: http://www.redalyc.org/articulo.oa?id=59335809004

Cómo citar el artículo

- Número completo

- Más información del artículo

- Página de la revista en redalyc.org

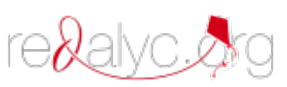

Sistema de Información Científica Red de Revistas Científicas de América Latina, el Caribe, España y Portugal Proyecto académico sin fines de lucro, desarrollado bajo la iniciativa de acceso abierto 


\title{
PALANCA RETRÁCTIL DE BAJO COSTO PARA INTERFACES CON PUERTO PARALELO Y VISUAL BASIC ${ }^{\circledR}$
}

\author{
LOW-COST RETRACTABLE LEVER FOR PARALLEL PORT \\ INTERFACES WITH VISUAL BASIC®
}

ROGELIO ESCOBAR Y CARLOS A. PÉREZ-HERRERA

UNIVERSIDAD NACIONAL AUTÓNOMA DE MÉXICO

LABORATORIO DE CONDICIONAMIENTO OPERANTE

\begin{abstract}
Resumen
Se describe la construcción de palancas retractiles usando los arreglos de motores a pasos, tornillos sin fin y controladores para motores que se encuentran dentro de las unidades de disco de 3.5 pulgadas (floppy disk). Estas unidades que actualmente están en desuso pueden encontrarse con relativa facilidad en computadoras desechadas con un bajo costo. Adicionalmente se describen las conexiones para controlar dichas palancas retráctiles por medio de una interfaz con un puerto paralelo usando programación en Visual Basic $®$ Express Edition.

Palabras clave: palanca retráctil, interfaz con puerto paralelo, Visual Basic, instrumentación
\end{abstract}

\begin{abstract}
This paper describes the construction of retractable levers using the array of a stepper motor, a worm drive, and a motor controller that can be found as part of the mechanism of 3.5" floppy disk reader. These inexpensive and now obsolete disk readers can be found with relative ease in old computers. The paper describes the electrical connections used to control the levers by means of a parallel port interface with Visual Basic ${ }^{\circledR}$ Express Edition programming.

Keywords: retractable lever, parallel port interface, Visual Basic, instrumentation

Rogelio Escobar y Carlos A. Pérez-Herrera, Laboratorio de Condicionamiento O perante, Facultad de Psicología, Universidad Nacional Autónoma de México.

Este trabajo se realizó con el apoyo del proyecto TA300213-2 otorgado por el PAPIIT de DGAPA, UNAM.

La correspondencia relacionada con este artículo debe enviarse a Rogelio Escobar, Laboratorio de Condicionamiento O perante, 2ํ Piso, Edificio C. Facultad de Psicología, U niversidad Nacional Autónoma de México. Av. Universidad 3004, Col. Copilco-U niversidad, M éxico, D.F. C.P. 04510. E-mail: rescobar@unam.mx.
\end{abstract}


Las palancas retráctiles son dispositivos para el registro de respuestas comúnmente empleadas en el análisis experimental de la conducta cuando se usan roedores como sujetos en experimentos en los que se requiere que la palanca no esté disponible continuamente. Aunque han existido dispositivos de respuesta para ratas que evitan automáticamente que la respuesta blanco ocurra durante ciertos periodos de tiempo, por ejemplo al bloquear el movimiento de una puerta (e.g., Skinner, 1930), una vez que se adoptaron las palancas como dispositivos de respuesta (e.g., Skinner, 1932), las palancas que pueden introducirse o retraerse de la cámara experimental se han vuelto la regla en estas preparaciones experimentales.

En la década de 1960 aumentaron notablemente los procedimientos en los cuales se requería el uso de palancas retractiles y se diseñaron palancas que podían fabricarse por los experimentadores (Hineline, 1968; Hoffman, 1964; H uneycutt, Crowder, \& Wilkes, 1964). Estas palancas de extensión y retracción automática sustituyeron la retracción manual de la palanca que se reportó en algunos trabajos anteriores (e.g., Denny, 1959; Skinner 1938, p. 92). Algunos ejemplos notables del uso de las palancas retráctiles con ratas como sujetos son los procedimientos de ensayo discreto, los programas concurrentes encadenados y los procedimientos de elección inter temporal.

Existen diversos modelos de palancas retráctiles que son distribuidas comercialmente por compañías como Med Associates o Coulbourn. Sin embargo, la palanca retráctil que se describe en este trabajo tiene las ventajas de que puede construirse con un bajo costo usando componentes que pueden encontrarse fácilmente en computadoras de desecho y es compatible con la interfaz que utiliza el puerto paralelo de una computadora y se controla por medio de Visual Basic Express Edition (VBEE) descrita en trabajos anteriores (Escobar \& Lattal, 2010; Escobar, Hernández-Ruiz, Santillán, \& Pérez-Herrera, 2012). La palanca retráctil descrita en este trabajo utiliza el mecanismo que se encuentra dentro de una unidad lectora de discos magnéticos de 3.5 pulgadas (floppy disk). Estas unidades pueden conseguirse nuevas por al rededor de 100 pesos mexicanos y usadas por alrededor de 50 pesos mexicanos. Para las pruebas se usaron dos unidades de disco marca NEC modelo FD $1231 \mathrm{H}$ pero el diseño se probó exitosamente con diversas marcas y modelos.

\section{Construcción}

El primer paso en la construcción consistió en desmontar la cubierta de una unidad lectora de discos magnéticos de 3.5 pulgadas. En caso de que necesiten usarse dos o más palancas es importante que las unidades de disco sean idénticas debido a que el ruido que produce el motor y la velocidad de desplazamiento podría variar. Esto puede verificarse al comparar las etiquetas y la ubicación de los componentes en la parte trasera de la unidad de disco. Las partes mecánicas relacionadas con la entrada del disco y el motor que permite que el disco gire pueden eliminarse dejando espacio para colocar la palanca. En la parte superior de la Figura 1 se muestran tres 
unidades con la tapa removida y los elementos asociados con la entrada y rotación del disco eliminados. En la parte inferior de la figura se muestran las unidades por la parte de abajo donde se encuentran los circuitos. Es importante mantener intactos los circuitos que se encuentran en la parte posterior porque dentro de estos se encuentra el controlador del motor a pasos.

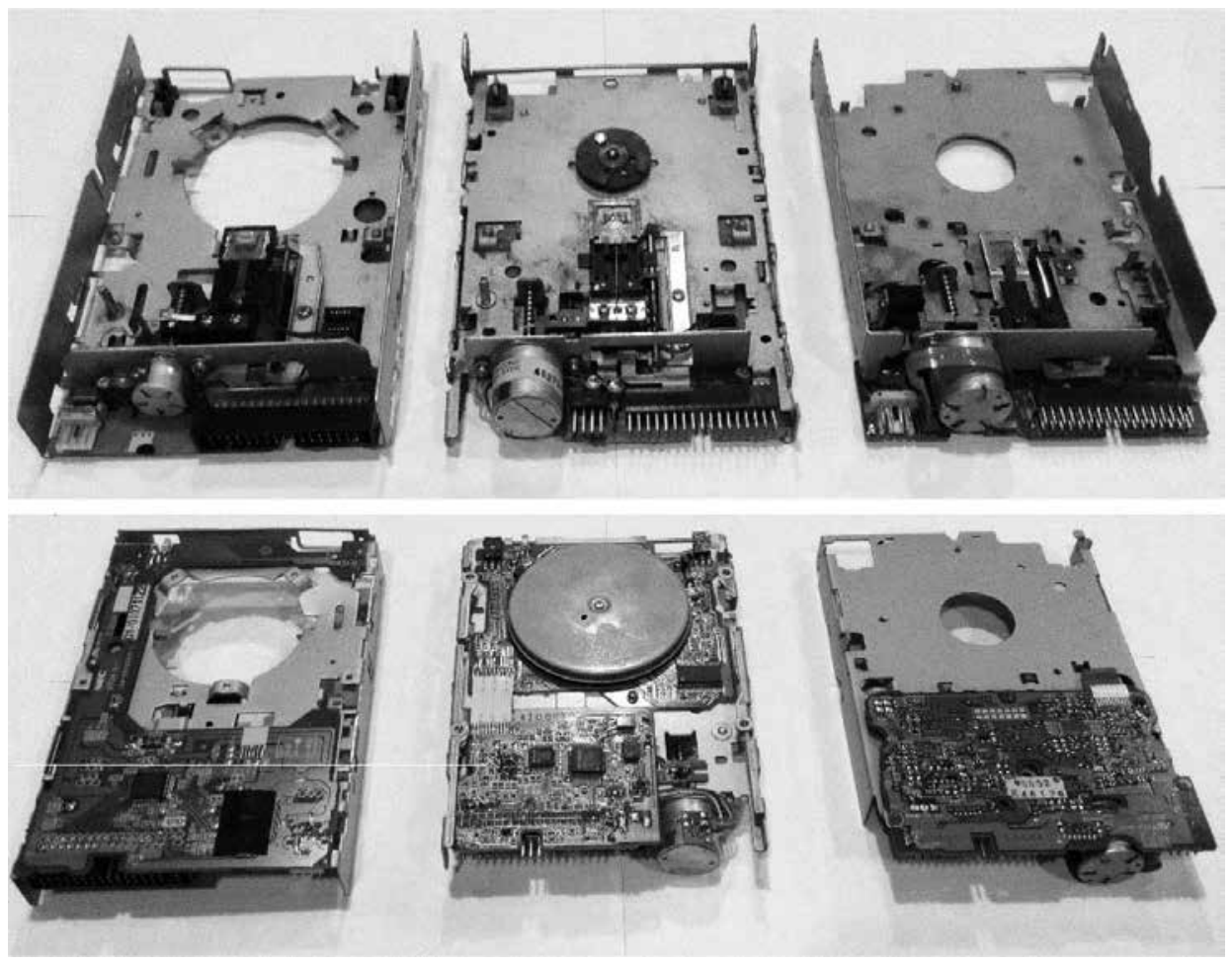

Figura 1. Vista posterior de tres modelos de unidad de disco de 3.5 pulgadas. El mecanismo que permite el acceso del disco fue removido para dejar al descubierto el mecanismo que permite el avance y el retroceso del motor a pasos. En la parte de arriba de la figura se muestra la vista superior de las unidades y en la parte de abajo se muestra una vista inferior de las unidades.

Una vez que el motor a pasos y el mecanismo de avance del motor quedan expuestos pueden identificarse los conectores y las partes que se utilizan para controlar las palancas retráctiles (véase la Figura 2). Se añadió un riel de aluminio para montar la palanca. Este riel se atornilló al soporte de plástico que está unido al tornillo 
sin fin. En las unidades que revisamos, aunque el soporte era de plástico, el orificio roscado para los tornillos era de metal. Se atornilló un micro interruptor (microswitch) de palanca larga en el riel después de verificar la distancia de la palanca relativa a la cámara experimental. Se añadió una lámina de metal al final de la lámina delgada del micro interruptor para aumentar la superficie de la palanca. La parte frontal de la caja de metal de la unidad de disco puede atornillarse a la cámara experimental. En nuestro diseño también usamos un ángulo de aluminio para evitar que la unidad se moviera y usamos dos unidades de disco para montar dos palancas. En la Figura 3 se muestran las dos palancas retráctiles montadas en la cámara experimental.

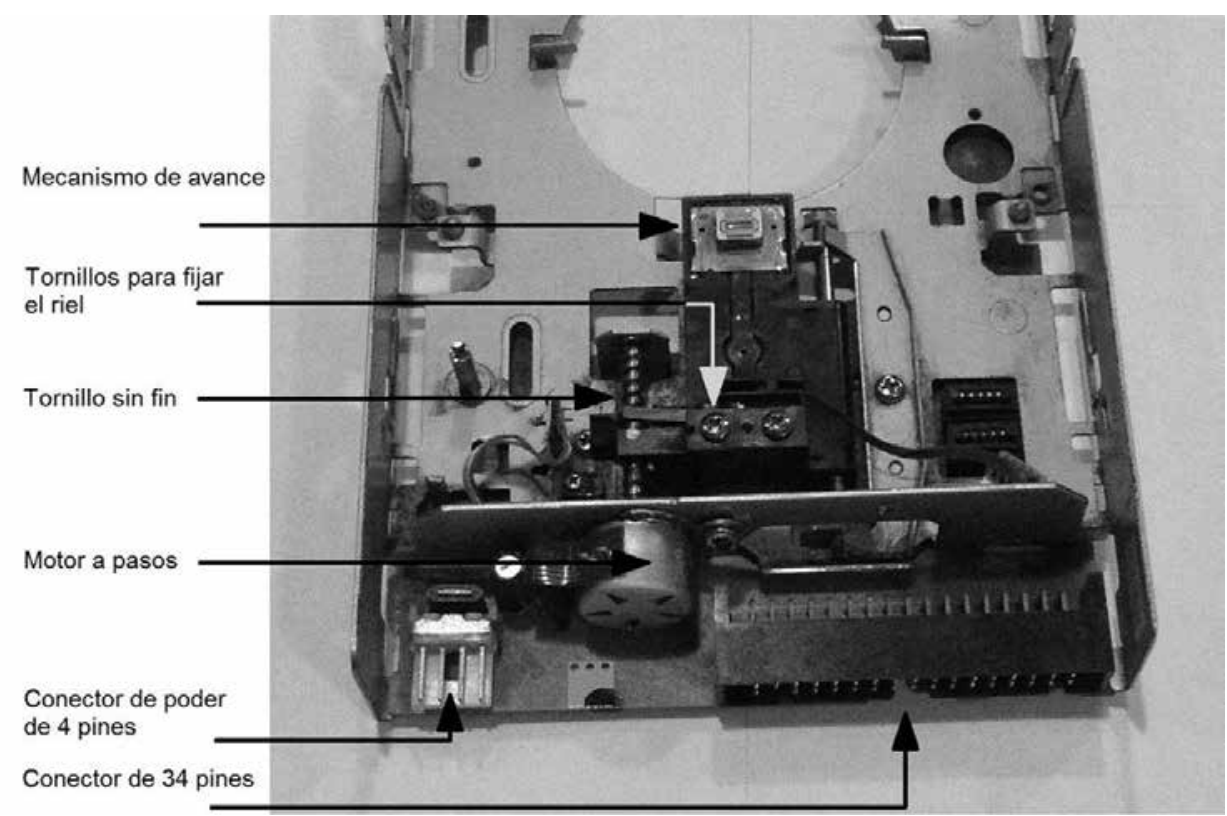

Figura 2. Parte posterior de la unidad de disco con los componentes necesarios para montar la palanca retráctil.

\section{Conexión}

Las conexiones eléctricas pueden hacerse en conjunto con la interfaz de puerto paralelo descrita en los trabajos de Escobar y Lattal (2010) y Escobar et al. (2012). Los pines del puerto de datos del puerto paralelo que se usan como salidas (outputs) permiten activar directamente el motor de la palanca retráctil. Este motor funciona con $+5 \mathrm{v}$ que pueden tomarse de cualquier cable rojo proveniente de la fuente de 


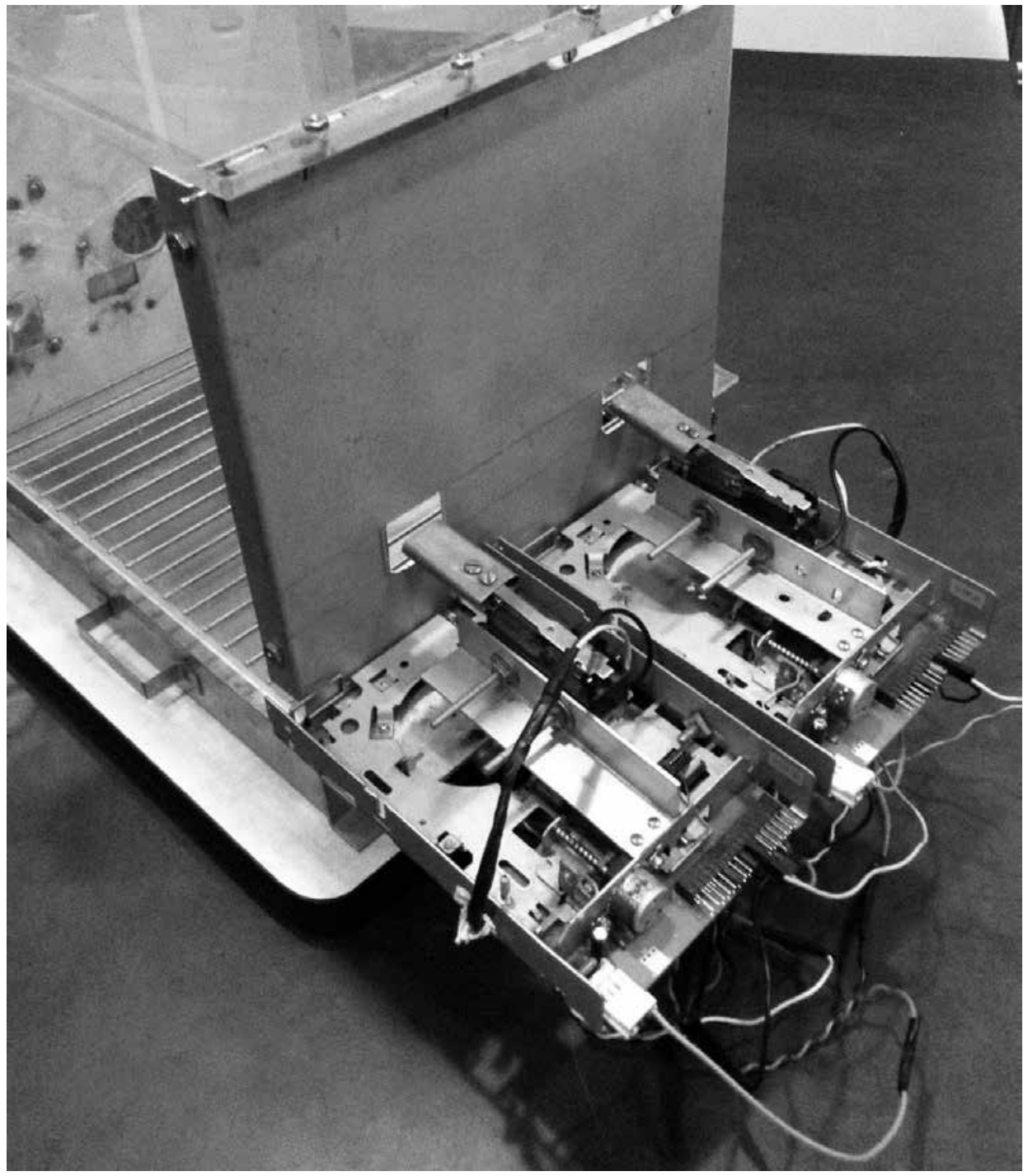

Figura 3. Palancas retráctiles montadas en la cámara de condicionamiento operante 
poder de la computadora o de una fuente de poder externa. El cable con $+5 \mathrm{v}$ se conecta al pin de la derecha del conector de poder de la unidad de disco (floppy power connector) que tiene cuatro pines. El cable a tierra (GND), que puede tomarse de cualquier cable negro de la fuente de poder de la computadora, se conecta a cualquiera de los dos pines centrales. En la Figura 4 se muestra el diagrama de las conexiones. Durante las pruebas descubrimos que el regreso del motor para retraer las palancas puede ser problemático y en algunas unidades de disco no ocurrió. Para evitar este problema se añadió un relevador de estado sólido (Panasonic PhotoMOS, Modelo AQ Z102) para cortar el suministro de corriente al motor, de esta forma la palanca se pudo retraer rápidamente en todas las unidades de disco que se probaron.

Con una sola palanca pueden usarse los pines 6 y 8 (puerto de datos $888 / 378 \mathrm{~h}$,

AQZ102

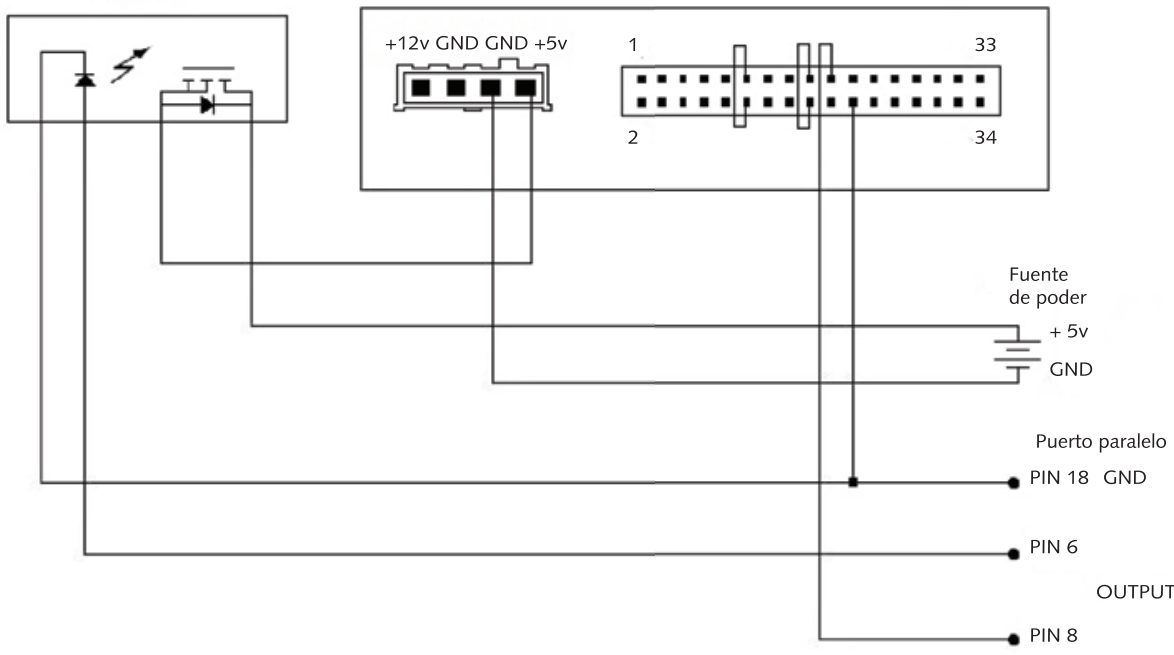

Figura 4. Diagrama de las conexiones del puerto paralelo y la fuente de poder de una computadora con el relevador de estado sólido (AQ Z102) y con los conectores de la unidad de disco. Los Pines 11 y 12 del conector de 34 pines y los Pines 17 y 18 se conectaron por medio de un mini jumper.

D 4 y D6) del puerto paralelo para controlar la retracción y la extensión de la palanca, respectivamente, y para una segunda palanca pueden usarse los pines 7 y 9 (puerto de datos 888/378h D 5 y D 7). De acuerdo con el diagrama, si se cambia el estado del 
Pin 6 (D 4) del puerto paralelo a encendido (high), se permite que +5 v lleguen al motor de la unidad de disco por medio del relevador. Una vez que el Pin 6 está encendido, al encender el Pin 8 (D6) se activa el motor y se extiende la palanca. Cuando se apaga el Pin 8, se corta la conexión entre el motor y la fuente de poder, y la palanca se retrae rápidamente.

\section{Programación}

La extensión y retracción de las palancas puede realizarse por medio de funciones en VBEE para encender y apagar los pines apropiados en el puerto paralelo. Escobar y Lattal (2010) y Escobar et al. (2012) describieron el código en VBEE que se utiliza para controlar la interfaz con el puerto paralelo. Como se describió en dichos artículos para controlar el puerto paralelo es necesario habilitar las funciones del archivo inpout32.dll como entrada y salida en VBEE (véase Escobar \& Lattal, p. 15). En el Apéndice se muestra el código para controlar las dos palancas que se usaron en este trabajo. Las funciones que se describen en el Apéndice pueden añadirse en cualquier momento durante el programa en el que se requiera la extensión y retracción de la palanca. Se añadió una explicación en cada una de las líneas del programa para facilitar su análisis y modificación. En este programa, las funciones se añadieron en un módulo independiente.

Durante las pruebas del equipo se enfrentaron algunos problemas. Un problema fue que las palancas extendidas únicamente se introdujeron $1.5 \mathrm{~cm}$ dentro de la cámara experimental. En comparación, las palancas comercializadas por M ed Associates se extienden $1.9 \mathrm{~cm}$. Esta diferencia podría ser importante en los experimentos pero es necesario realizar pruebas más extensas para determinarlo. 0 tro problema fue que en nuestra cámara experimental, originalmente la ranura para la palanca permitía que hubiera un espacio entre la palanca y la parte superior de la ranura. Debido a este espacio, al retraer la palanca, las ratas podían introducir la pata y presionar la palanca. Para evitar este problema es necesario no dejar espacio entre la ranura y la parte superior de la palanca. 0 tra forma de solucionar este problema sería añadir un mecanismo de bloqueo de la palanca cuando esta se encuentre retraída. Sin embargo, esta última opción no fue necesaria en nuestro equipo. A pesar de estos problemas, estas palancas retráctiles pueden acoplarse con relativa facilidad a la interfaz con el puerto paralelo, son de construcción simple y de bajo costo. Consideramos que estas ventajas las hacen una opción viable para sustituir a las palancas retráctiles comerciales.

\section{Referencias}

Denny, M. R. (1959). One bar-press a day: Acquisition and extinction. Journal of the Experimental Analysis of Behavior 2, 81-85. doi: 10.1901/jeab.1959.2-81

Escobar, R., Hernández-Ruiz, M., Santillán, N., \& Pérez-Herrera, C., (2012). Nota técnica: diseño simplificado de una interfaz de bajo costo usando un puerto para- 
lelo y visual basic. Revista Mexicana de Análisis de la Conducta, 38, 72-88.

Escobar, R., \& Lattal, K. (2010). Interfaz de bajo costo usando un puerto paralelo y visual basic. Revista Mexicana de Análisis de la Conducta, 36, 7-21.

Hineline P. N. (1968). A rapid retractable response lever. Journal of the Experimental Analysis of Behavior 11, 127-128. doi: 10.1901/jeab.1968.11-127

Hoffman, H. S. (1964). A retractable lever for use in behavioral research. Journal of the Experimental Analysis of Behavior 7, 163-164. doi: 10.1901/jeab.1964.7-163

Huneycutt, B. D., Crowder, W. F., \& Wilkes, W. P. (1964). An inexpensive, retracting rat lever. Journal of the Experimental Analysis Behavior, 7, 332. doi: 10.1901/jeab.1964.7-332

Skinner, B. F. (1930). The concept of the reflex in the description of behavior. Doctoral dissertation, Harvard University. Cambridge, MA.

Skinner, B. F. (1932). Drive and reflex strength: II. Journal of General Psychology, 6, 38-48. Skinner, B. F. (1938). The behavior of organisms: An experimental analysis. Nueva York, Estados Unidos: Appleton-Century-Crofts.

\section{Apéndice}

\section{Module RetractableLevers}

Dim turn As Integer 'Esta variable cuenta el número de activaciones del motor y permite controlar el número de vueltas de éste

Function Motor10 n() As Integer 'Esta función extiende la Palanca 1 al encender y apagar alternadamente el motor

For turn $=1$ To 87 'Son necesarias 87 activaciones del motor para que alcance la máxima extensión

System.Threading.Thread.Sleep(1) 'Pausa necesaria para que se alterne el encendido y el apagado del motor

O ut $(888,80$ ) 'Enciende el Pin 6 (Bin 16) y 8 (Bin 64). Esto es, permite el paso de $+5 \mathrm{v}$ al Motor 1 y lo activa

System.Threading.Thread.Sleep(1) 'Pausa necesaria para que se alterne el encendido y el apagado del motor

nuevamente

O ut(888, 16) 'Enciende el Pin 6 (Bin 16) para que esté listo para activarse

N ext turn

End Function

Function M otor20 n() As Integer 'Esta función extiende la Palanca 2

For turn $=1$ To 87

System.Threading.Thread.Sleep(1)

O ut(888, 160) 'Enciende el Pin 7 (Bin 32) y 8 (Bin 128). Esto es, permite el 
paso de $+5 v$ al Motor 2 y lo activa

System.Threading.Thread.Sleep(1)

O ut(888, 32) 'Enciende el Pin 7 (Bin 32) para que esté listo para activarse nuevamente

Next turn

End Function

Function Motor10 ff() As Integer 'Esta función retrae la Palanca 1

O ut(888, 0) 'A paga to dos Ios pines del puerto paralelo

System.Threading.Thread.Sleep(4)

O ut $(888,16)^{\prime}$ Enciende el Pin 6 (Bin 16)

System.Threading.Thread.Sleep(1)

End Function

Function M otor20 ff() As Integer 'Esta función retrae la Palanca 2

O ut $(888,0)$

System.Threading.Thread.Sleep(4)

O ut(888, 32) 'Enciende el Pin 7 (Bin 32)

System.Threading.Thread.Sleep(1)

End Function

Function MotorsO n() As Integer 'Esta función extiende las dos palancas

For turn = 1 To 87

System.Threading.Thread.Sleep(1)

Pin 9 (Bin 128)

O ut(888, 240) 'Enciende el Pin 6 (Bin 16), Pin 7 (Bin 32), Pin 8 (Bin 64) y

System.Threading.Thread.Sleep(1)

O ut $(888,48)$ 'Enciende el Pin 6 (Bin 16) y el Pin 7 para que estén listos para

activarse nuevamente

Next turn

End Function

Function M otorsO ff() As Integer 'Esta función retrae las dos palancas

O ut $(888,0)$

System.Threading.Thread.Sleep(4)

O ut $(888,48)$

System.Threading.Thread.Sleep(1)

End Function

End Module 